\title{
Prognostic performance of age-adapted SOFA and qSOFA in septic children
}

\author{
Xuepeng Zhang ${ }^{1,2 \dagger}$, Xiying Gui ${ }^{1 \dagger}$, Kaiying Yang ${ }^{2}$, Siyuan Chen ${ }^{1^{*}}$ (D) and $\mathrm{Yi}_{\mathrm{Ji}^{2^{*}}}$
}

Dear editors:

The criteria used to define pediatric sepsis have not been updated for nearly 15 years since the establishment of the 2005 International Pediatric Sepsis Consensus. Some investigators adapted the Sepsis 3.0 criteria to pediatric sepsis definition [1].

Between January 2018 and July 2019, we prospectively enrolled 342 children from PICU (Clinicaltrials.gov, NCT03598127) with sepsis on admission according to the 2005 Pediatric Sepsis Consensus. Age-adapted SOFA and quick SOFA (qSOFA) were used as described in a previous study [1]. We assessed the performance of age-adapted SOFA and qSOFA, Pediatric Risk of Mortality (PRISM), and pediatric logistic organ dysfunction (PELOD)-2 scores on predicting mortality among septic children by using the area under the receiver operating characteristic curve (AUROC).

The median age was 9 months, and 192 children (56.14\%) were boys (Table 1); 20 children died in hospital, with a mortality rate of almost $6 \%$. Performances of the four tools on discriminating survival are showed in Fig. 1: the PELOD-2 scores and PRISM scores had good discrimination $(0.871,95 \%$ CI $0.831-$ 0.905 and $0.868,95 \%$ CI $0.828-0.902$ respectively), bet- ter than age-adapted SOFA scores $(0.790,95 \%$ CI 0.743-0.832). Age-adapted qSOFA had the smallest AUROC (0.639, 95\% CI 0.586-0.690).

Our data revealed that age-adapted qSOFA may not be a good predictor of mortality for pediatric sepsis. Quick SOFA has a range from 0 to 3 . It is possible that four variations may be insufficient to describe various severity of sepsis. In addition, scoring of Glasgow coma scale (GCS) score may be inaccurate in children, especially in young infants.

Age-adapted qSOFA may be sensitive in recognizing patients with sepsis and facilitates clinicians to quickly find out children who are at high risk for sepsis. However, when considering the limited specificity, promoting qSOFA in children with sepsis may have little benefit. It was reported that the mortality in pediatric sepsis was $3.5-4.4 \%[2,3]$, which is similar to or even lower than the general mortality in PICU $[4,5]$ but much lower than the mortality in adult sepsis. Should we urge to adapt qSOFA to pediatric sepsis, in which the mortality is not higher than that in general ICU population? Maybe we could focus our attention on the patients with septic shock in whom the mortality is higher than $30 \%$, as this may be more helpful to improve outcomes in children with sepsis.

\footnotetext{
* Correspondence: siy_chen@163.com; jijiyuanyuan@163.com

${ }^{\dagger}$ Xuepeng Zhang and Xiying Gui are authors contributed to the work equally and should be regarded as co-first authors.

${ }^{1}$ Department of Critical Care Medicine, West China Hospital of Sichuan

University, Chengdu 610041, China

${ }^{2}$ Department of Pediatric Surgery, West China Hospital of Sichuan University,

\#37 Guo-Xue-Xiang, Chengdu 610041, Sichuan, China
}

(c) The Author(s). 2019 Open Access This article is distributed under the terms of the Creative Commons Attribution 4.0 International License (http://creativecommons.org/licenses/by/4.0/), which permits unrestricted use, distribution, and reproduction in any medium, provided you give appropriate credit to the original author(s) and the source, provide a link to the Creative Commons license, and indicate if changes were made. The Creative Commons Public Domain Dedication waiver (http://creativecommons.org/publicdomain/zero/1.0/) applies to the data made available in this article, unless otherwise stated. 
Table 1 Baseline characteristics

\begin{tabular}{ll}
\hline Characteristics & Value \\
\hline Age, months & $9.00(2.75,42.0)$ \\
Male, $n(\%)$ & $192(56.14 \%)$ \\
Source of infection, $n(\%)$ & \\
Respiratory system & $187(54.68 \%)$ \\
Gastrointestinal system & $81(23.68 \%)$ \\
Central nervous system & $13(3.8 \%)$ \\
Bloodstream & $33(9.65 \%)$ \\
Other & $28(8.19 \%)$ \\
Length of ICU stay, days & $7.90(4.50,13.60)$ \\
PRISM & $8.0(6.0,13.0)$ \\
PELOD-2 & $4.0(2.0,6.0)$ \\
qSOFA & $2.0(1.0,3.0)$ \\
SOFA & $4.0(3.0,7.0)$ \\
Mortality, $n$ (\%) & $20(5.85 \%)$ \\
\hline
\end{tabular}

Data are presented as median (interquartile range) or number (percentage)

Abbreviations: PICU pediatric intensive care unit, PRISM Pediatric Risk of

Mortality, PELOD-2, pediatric logistic organ dysfunction-2, qSOFA quick

Sequential Organ Failure Assessment, SOFA Sequential Organ

Failure Assessment

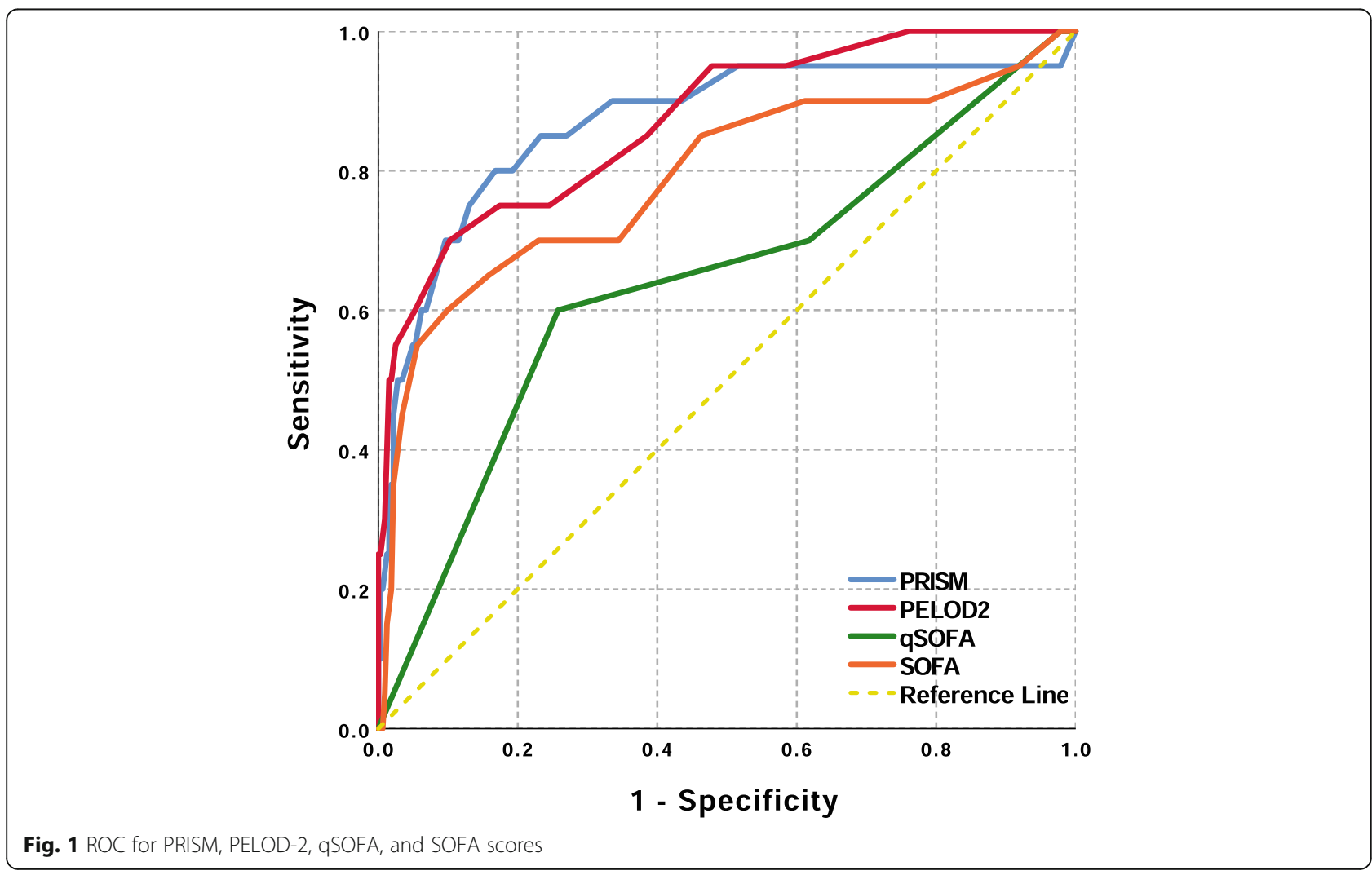




\section{Abbreviations}

PICU: Pediatric intensive care unit; PRISM: Pediatric Risk of Mortality: PELOD: Pediatric logistic organ dysfunction; SOFA: Sequential Organ Failure Assessment; qSOFA: Quick Sequential Organ Failure Assessment;

Cl: Confidence interval; AUROC: Area under the receiver operating characteristic curve; GCS: Glasgow coma scale

\section{Acknowledgements}

We would like to thank the parents of our patient for their cooperation, and also thank the staff for the collection of the data.

\section{Authors' contributions}

SYC, YJ, and XPZ designed the study. XPZ and KYY managed the data and its quality. XPZ and XYG performed the statistical analysis and drafted the manuscript. All authors participated in the data interpretation. YSC and YJ contributed substantially to its revision. All authors read the manuscript carefully and approved the final version.

\section{Funding}

This project was supported by grants from the National Natural Science Foundation of China (Grants 81400862 and 81401606), the Key Project in the Science \& Technology Program of Sichuan Province (Grant No:

2019YFS0322), and the Science Foundation for The Excellent Youth Scholars of Sichuan University (Grants 2015SU04A15).

\section{Availability of data and materials}

The datasets used for the analysis in the current study are available from the corresponding author on reasonable request.

\section{Ethics approval and consent to participate}

The study was approved by the Ethics Committee of the West China Hospital of Sichuan University. Informed consents were obtained from the legal guardians of the children included in the study.

\section{Consent for publication}

Not applicable.

\section{Competing interests}

The authors declare that they have no competing interests.

Received: 26 August 2019 Accepted: 13 September 2019

\section{Published online: 29 October 2019}

\section{References}

1. Schlapbach LJ, Straney L, Bellomo R, MacLaren G, Pilcher D. Prognostic accuracy of age-adapted SOFA, SIRS, PELOD-2, and qSOFA for in-hospital mortality among children with suspected infection admitted to the intensive care unit. Intensive Care Med. 2018;442:179-88.

2. Wang Y, Sun B, Yue H, Lin X, Li B, Yang X, et al. An epidemiologic survey of pediatric sepsis in regional hospitals in China. Pediatr Crit Care Med. 2014; 159:814-20.

3. de Souza DC, Shieh HH, Barreira ER, Ventura AM, Bousso A, Troster EJ. Epidemiology of sepsis in children admitted to PICUs in South America. Pediatr Crit Care Med. 2016;178:727-34.

4. Leteurtre S, Grandbastien B, Leclerc F, Parslow R. International comparison of the performance of the paediatric index of mortality (PIM) 2 score in two national data sets. Intensive Care Med. 2012;388:1372-80.

5. Kim SY, Kim S, Cho J, Kim YS, Sol IS, Sung Y, et al. A deep learning model for real-time mortality prediction in critically ill children. Crit Care. 2019;231:279.

\section{Publisher's Note}

Springer Nature remains neutral with regard to jurisdictional claims in published maps and institutional affiliations. 